\title{
Universal Structural Mechanics
}

\section{Paul TE Cusack*}

Independent Researcher, BSc E, DULE, 1641 Sandy Point Rd, Saint John, NB, Canada E2K 5E8, Canada

\begin{abstract}
In this paper, we provide some interesting spot calculations from the Theory of Elastic Stability. Certain key constants of Cusack's Astrotheology are derived from basic structural mechanics' formula. No attempts for proofs are made. The reader is referred to Timoshenko and Gere's classic book on stability. Proton Mass was determined to be within the margin of error.
\end{abstract}

Keywords: Elastic stability; Astrotheology; Moment; Euler's critical load; Golden mean parabola

\section{Introduction}

Consider the beam column. Using our knowledge of Physical Constants derived in Astrotheology math, we can derive other variable, such as energy and time. The Super force is sin t. we begin with a column loaded with that Super force load. Then we move on to a beam column loaded laterally as well as axially. We end with Euler's Critical load which allows us to derive the maximum mass in the periodic table of the elements (Figure 1).

Refer to Figure 1 showing cusack's modulus $\mathrm{k}=\mathrm{cuz}=0.4233$.

$\mathrm{y}=\sin \theta$

$y^{\prime}=-\cos \theta$

$0.4233=\mathrm{cuz}=-\cos \theta$

$\theta=64.95^{\circ}=113.37 \mathrm{rads}$

$=1 / 0.882 \sim 1 / \varepsilon_{0}$

And,

$\theta=64.95^{\circ}=1 / 0.1539=1 /(1-\sin 1.0085 \mathrm{rads})$

$1.0085=$ Mass of $\mathrm{H}^{+}$

Continuing,

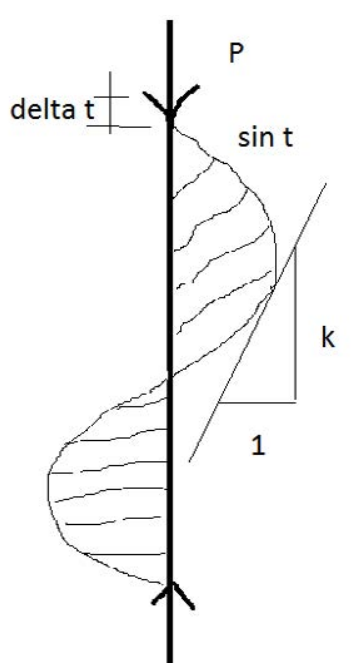

Figure 1: Beam column showing $\mathrm{k}$.

$$
\begin{aligned}
& \int_{0-2 \pi} \sin \theta=|-\cos \theta| \\
& -(-1)-(1)=2=-\mathrm{dM} / \mathrm{dt}
\end{aligned}
$$

$\Delta \mathrm{t} / \mathrm{dt}=1 \mathrm{rad} / 1 \mathrm{sec}$.

$\sin 1=0.8415=1 / 118=1 / \mathrm{M}$ (Periodic Table of the elements)

EI $d^{3} y / d x^{3}+P d y / d x=-V[1]$ pg. 2

$(0.4233) \mathrm{I}(\mathrm{G})+2.667(0.8415)=-1.617$

$\mathrm{I}=0,4936$

$1 / \mathrm{I}=\mathrm{Y}=0.202$

$\mathrm{I}=1 / \mathrm{Y}$ where $\mathrm{Y}=\mathrm{e}^{-\mathrm{t}} \cos (2 \pi \mathrm{t}) \& \mathrm{t}=1$

EI $\mathrm{d}^{2} \mathrm{M} / \mathrm{dx}^{2}=-\mathrm{M}[1]$.

(0.4233) $(1 / \mathrm{Y})(0.8415)=\mathrm{M}=1758=1.00735 \sim \mathrm{Mass} \mathrm{H}^{+}$(Figure 2).

$\mathrm{d}^{2} \mathrm{y} / \mathrm{dx}^{2}+\mathrm{k}^{2} \mathrm{y}=-\mathrm{Qc} \mathrm{x} /$ [EIl] [1]

Aside:

$\mathrm{k}^{2}=\mathrm{P} /[\mathrm{EI}]$

$=2.7667 /(1 / 0.4233)(1 / \mathrm{Y})$

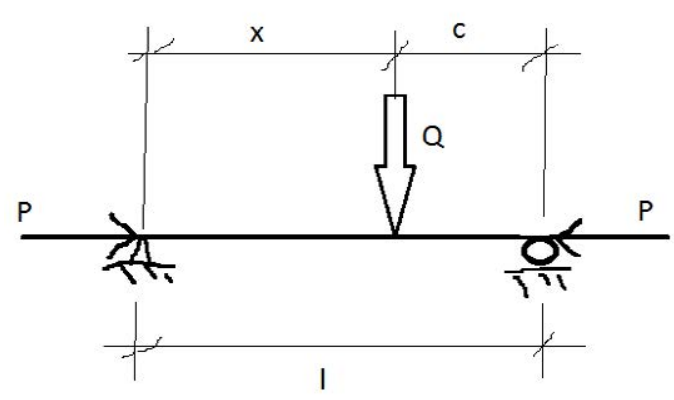

Figure 2: Beam column showing $Q$.

*Corresponding author: Cusack PTE, Independent Researcher, BSc E, DULE 1641 Sandy Point Rd, Saint John, NB, Canada E2K 5E8, Canada, Tel: (506) 2143313; E-mail: st-michael@hotmail.com

Received May 30, 2017; Accepted August 16, 2017; Published August 24, 2017

Citation: Cusack PTE (2017) Universal Structural Mechanics. Fluid Mech Open Acc 4: 173. doi: 10.4172/2476-2296.1000173

Copyright: @ 2017 Cusack PTE. This is an open-access article distributed under the terms of the Creative Commons Attribution License, which permits unrestricted use, distribution, and reproduction in any medium, provided the original author and source are credited. 


$$
=127.6
$$$$
=\rho
$$$$
-2.667 \mathrm{c} /[0.4233 \times(1 / \mathrm{Y}) \mathrm{l}] \mathrm{x}
$$$$
6.67+127.6=127.6 \mathrm{cx} / 1
$$$$
\mathrm{cx} / \mathrm{l}=522.5
$$

From the Golden mean parabola with roots -0.618 \& 1.618

$$
(-0.618) \mathrm{x} / \mathrm{l}=522.5
$$$$
\mathrm{x} / \mathrm{l}=118.3=\text { Mass } \mathrm{M}
$$$$
118.3=\mathrm{x} /(1.618+0.618)
$$$$
=\mathrm{x} / 224
$$$$
\mathrm{X}=52.91=1.083 \mathrm{rads}
$$$$
522.5=\mathrm{cx} / \mathrm{l}
$$$$
522=c \times 52.91) / 224
$$$$
\mathrm{c}=221
$$

$\mathrm{G}+127.6=2.667 \mathrm{cx} /[(0.4233)(1 / \mathrm{Y})(\mathrm{l})$

$\mathrm{Y}=202$

$6.67-1.27=2.667 / 0.4233 \times x / 0.202$

$2.667 / 0.4233=6.3=1$

$\mathrm{Y}(\mathrm{G}-\rho)=\mathrm{c}(1-\mathrm{c})$

$\mathrm{Y}(\mathrm{G}-\rho)=\mathrm{c}-\mathrm{c}^{2}$

Golden Mean parabola

$c^{2}-c+Y(G-\rho)=0$

$\mathrm{Y}(\mathrm{G}-\rho)=1$

$0.202(6.67-1.27)=1$

$24.5-1=23.6=\operatorname{Ln} \pi$.

Consider,

$\mathrm{y}=\mathrm{Q} \sin \mathrm{k}(\mathrm{l}-\mathrm{c}) /[\mathrm{P} \mathrm{k} \sin (\mathrm{kl})] \sin \mathrm{k}(\mathrm{l}-\mathrm{x})-\mathrm{Q}(\mathrm{l}-\mathrm{c})(\mathrm{l}-\mathrm{x}) / \mathrm{Pl}$

$$
\text { Let }
$$

$\mathrm{Q}=\mathrm{P}$

$\mathrm{k}=\sqrt{ } \rho$

$y=\sin 59.65 /[\sqrt{ } 127.6 \times \sin 253]-522.531$

$\mathrm{y}=-0.868$

$\mathrm{y}=\sin 60^{\circ}$

$\mathrm{Y}=0.868=\sin 60^{\circ}$

$\mathrm{Y}=\sqrt{ } 3 / 2$

$\mathrm{Y}=\mathrm{t} /[\mathrm{dM} / \mathrm{dt}]$

$\mathrm{dM} / \mathrm{dt}=\mathrm{t} / \mathrm{Y}$

$\mathrm{Y}=\mathrm{E}=\mathrm{dM} / \mathrm{dt} \times \mathrm{t}$

But $\mathrm{E}=\mathrm{Mc}^{2}$

$\mathrm{E}^{2} / 2=\mathrm{M} \mathrm{t}^{2} / 2$
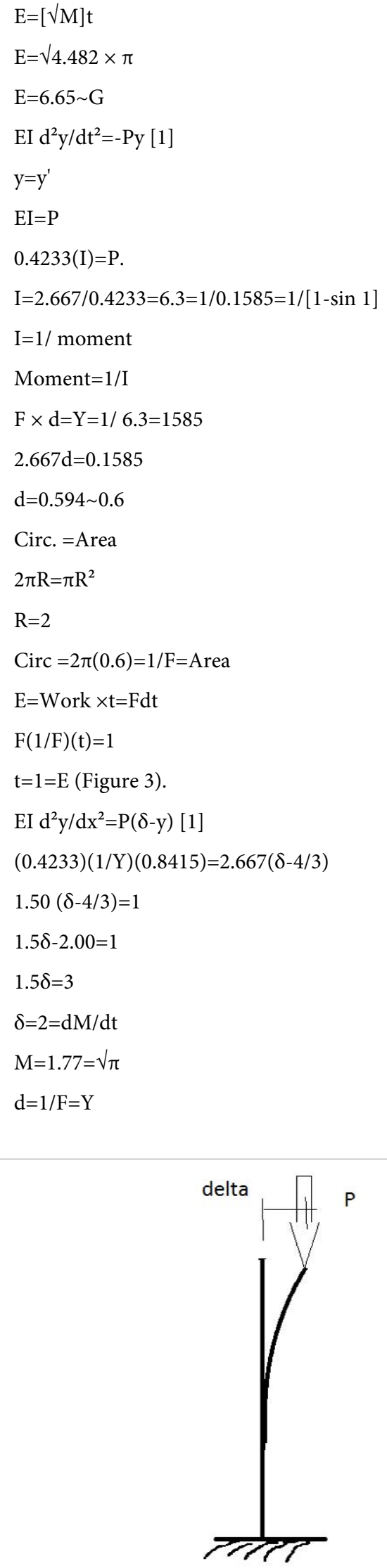

Figure 3: Eccentric load on column 


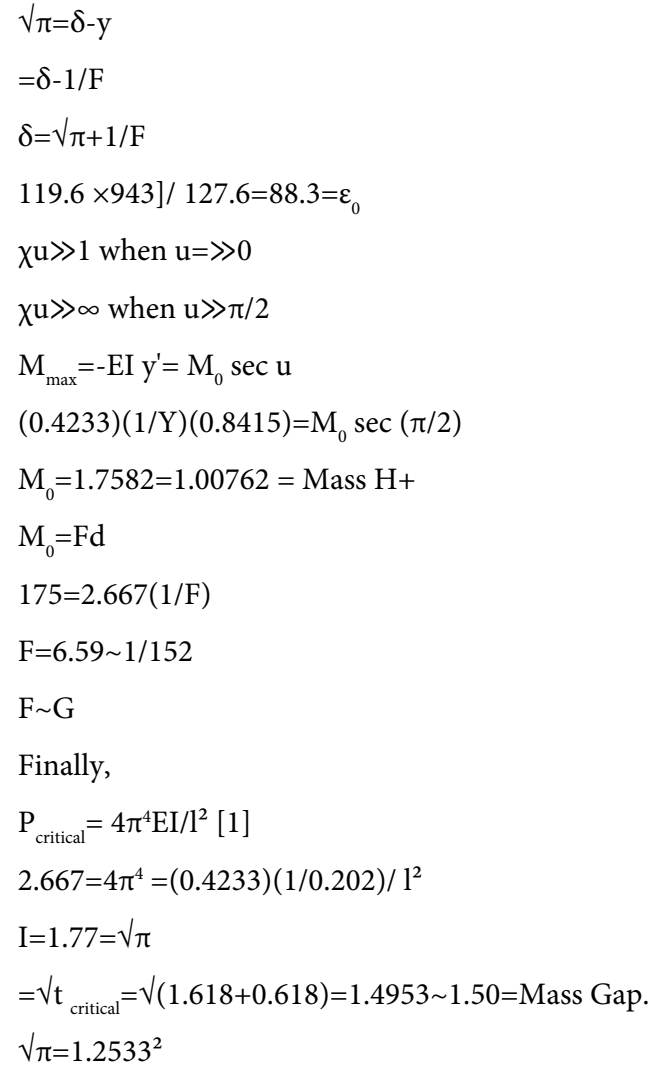

The rigidity of the universe is the density, and the critical load is the Mass.

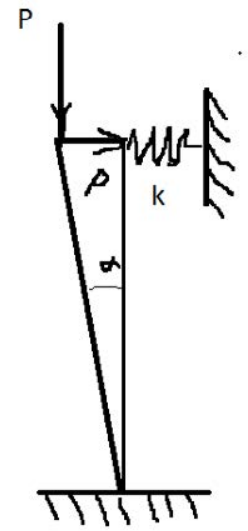

Figure 4: Proton mass column.

EI d $d^{4 y} / d x^{4}+P d^{3} y / d x^{3}+q / g d^{2} y^{2} / d x^{2}=0[1]$

$y=y^{\prime \prime}$ etc.

$\mathrm{EI}+\mathrm{P}+\mathrm{q} / \mathrm{G}=0$

$\mathrm{EI}+\mathrm{F}=\mathrm{G}[\mathrm{dM} / \mathrm{dx}]$.

\section{Mass-Gravity Equation [2]}

$\mathrm{dM} / \mathrm{dx}=[\mathrm{EI}+\mathrm{F}] / \mathrm{G}=[(0.4233)(6.3)+2.6678] / 6.67=0.799 \sim 0.8 \propto 8$

$1 / 8=1.25=\mathrm{E}_{\min }$

$\mathrm{M}=1 / \mathrm{c}^{4}=1 / 81=0.12345679$ Eight digits

Now Integrate wrt $\mathrm{x}$ or s:

$\int \mathrm{dM} / \mathrm{dx} d \mathrm{x}=1 / \mathrm{G}\left[\int \mathrm{EI} \mathrm{dx}+\int \mathrm{F} d \mathrm{x}\right]$

$\mathrm{M}=1 / \mathrm{G} \times \mathrm{E} 4 \mathrm{~s}^{5} / 5+\int \sin 60^{\circ}$

$\mathrm{M}=1 / 6.67 \times\left[(0)(0.4233)(4 / 3)^{5} / 5-\cos 60^{\circ}\right]$

$=0.75=3 / 4$

$\mathrm{M}=1 / \mathrm{s}$

$\mathrm{Ms}=\mathrm{Y}$

$\mathrm{Ms}=\mathrm{Fd} \times \mathrm{t}$

$\mathrm{dM} / \mathrm{dt} \times \mathrm{ds} / \mathrm{dt}=\mathrm{dF} / \mathrm{dt} \times \mathrm{dd} / \mathrm{dt}+\mathrm{dt} / \mathrm{dt}+\mathrm{C} 1$

$2(.8415)=(0.5)(0.8415)(1)+\mathrm{C} 1$

$1683=0.4208+\mathrm{C} 1$

$\mathrm{C} 1=126=\rho$

$1683-42=1641$

$1641+1 / c=1641+33.3333=1674$

$=1671.6+0.901$

$=\mathrm{Mp}++\mathrm{e}$.

\section{Conclusion}

In this paper, we provided some spot calculations using formula from elasticity stability theory. The beam column and the eccentricity 
loaded column can be used as a model for certain universal calculations. The rigidity of the universe is the density, and the critical load is the Mass. Perhaps those interested could find more in stability theory to explain Cusack's model of the Universe.

\section{References}

1. Timoshenko SP, Gere JM (2008) Theory of Elastic Stability (2ndedn), Dover New York.

2. Cusack P (2016) Astro-Theology, Cusacks Universe. J Phys Math 7: 174. 